\title{
Article \\ Somatic Disease in Survivors of Childhood Malignant Bone Tumors in the Nordic Countries
}

\author{
Camilla Pedersen ${ }^{1, *}$, Catherine Rechnitzer ${ }^{2}$, Elisabeth Anne Wreford Andersen ${ }^{1}$, Line Kenborg ${ }^{1}(\mathbb{D}$, \\ Filippa Nyboe Norsker ${ }^{1}{ }^{(0)}$, Andrea Bautz ${ }^{1}$, Thomas Baad-Hansen ${ }^{3}$, Laufey Tryggvadottir ${ }^{4,5}$, \\ Laura-Maria Madanat-Harjuoja ${ }^{6}$, Anna Sällfors Holmqvist ${ }^{7,8}$, Lars Hjorth ${ }^{7,8}$, Henrik Hasle ${ }^{9}$, \\ Jeanette Falck Winther ${ }^{1,10}$ and $^{-}$on behalf of the ALiCCS Study Group ${ }^{\dagger}$
}

Citation: Pedersen, C.; Rechnitzer, C.; Andersen, E.A.W.; Kenborg, L.; Norsker, F.N.; Bautz, A.;

Baad-Hansen, T.; Tryggvadottir, L.; Madanat-Harjuoja, L.-M.; Holmqvist, A.S.; et al. Somatic Disease in Survivors of Childhood Malignant Bone Tumors in the Nordic Countries. Cancers 2021, 13, 4505. https:// doi.org/10.3390/cancers13184505

Academic Editors: Saurabh Agarwal and Jianhua Yang

Received: 10 August 2021

Accepted: 3 September 2021

Published: 7 September 2021

Publisher's Note: MDPI stays neutral with regard to jurisdictional claims in published maps and institutional affiliations.

Copyright: (c) 2021 by the authors. Licensee MDPI, Basel, Switzerland. This article is an open access article distributed under the terms and conditions of the Creative Commons Attribution (CC BY) license (https:/ / creativecommons.org/licenses/by/ $4.0 /)$.
1 Danish Cancer Society Research Center, 2100 Copenhagen, Denmark; elian@cancer.dk (E.A.W.A.); kenborg@cancer.dk (L.K.); fnorsker@hotmail.com (F.N.N.); andrea.bautz@gmail.com (A.B.); jeanette@cancer.dk (J.F.W.)

2 Department of Pediatrics and Adolescent Medicine, Copenhagen University Hospital, 2100 Copenhagen, Denmark; catherine.rechnitzer@regionh.dk

3 Department of Orthopedic Surgery, Sarcoma Centre of Aarhus University Hospital, 8200 Aarhus, Denmark; thombaad@rm.dk

4 The Icelandic Cancer Registry, 105 Reykjavik, Iceland; laufeyt@krabb.is

5 Faculty of Medicine, University of Iceland, 102 Reykjavik, Iceland

6 The Finnish Cancer Registry, 00130 Helsinki, Finland; Laura.Madanat@cancer.fi

7 Department of Clinical Sciences Lund, Lund University, 22241 Lund, Sweden; anna.sallfors-holmqvist@med.lu.se (A.S.H.); Lars.Hjorth@skane.se (L.H.)

8 Department of Pediatric Hematology and Oncology, Skane University Hospital, 22185 Lund, Sweden

9 Department of Pediatrics, Aarhus University Hospital, 8200 Aarhus, Denmark; hasle@dadlnet.dk

10 Department of Clinical Medicine, Faculty of Health, Aarhus University and University Hospital, 8200 Aarhus, Denmark

* Correspondence: camped@cancer.dk

+ The ALiCCS Study Group consists of all researchers, data managers and clinicians who have been involved in setting up the large Nordic cohort of childhood cancer survivors within the research program 'Adult Life after Childhood Cancer in Scandinavia' (ALiCCS). Further, the group comprises an oversight Board composed of three cancer epidemiologists and three pediatric oncologists.

Simple Summary: The treatment of osteosarcoma and Ewing sarcoma, the two major types of malignant bone tumors in children, has progressed considerably during the last decades, with more patients becoming long-term survivors. This improvement has resulted in an increasing number of patients with long-term adverse health consequences from the life-saving treatment. The aim of this study was to provide a detailed, comprehensive overview of somatic diseases that require hospitalization in long-term survivors of osteosarcoma and Ewing sarcoma. This study contributes new insights into the risk of somatic late effects in survivors of osteosarcoma and Ewing sarcoma which are urgently requested by pediatric oncologists, researchers, and by survivors and their families. The study provides an essential basis for the development of preventive intervention strategies and for optimal patient counseling and follow-up care, which all contribute to improving the health and quality of life in survivors.

Abstract: Survivors of malignant bone tumors in childhood are at risk of long-term adverse health effects. We comprehensively reviewed cases of somatic diseases that required a hospital contact in survivors of osteosarcoma and Ewing sarcoma. In a population-based cohort study, 620 five-year survivors of osteosarcoma $(n=440)$ or Ewing sarcoma $(n=180)$, diagnosed before the age of 20 years in Denmark, Finland, Iceland, and Sweden during 1943-2008, were followed in the national hospital registers. Overall rates of hospital contacts for any somatic disease and for 12 main diagnostic groups and 120 specific disease categories were compared with those in a matched comparison cohort $(n=3049)$ randomly selected from the national population registers. The rate of hospital contact for any somatic disease was $80 \%$ higher in survivors of malignant bone tumors than in comparisons and remained elevated up to 30 years after diagnosis. The rate of hospital contacts was higher after Ewing sarcoma (rate ratio (RR) 2.24; 95\% confidence interval (CI) 1.76-2.85) than after osteosarcoma 
(RR 1.67; 95\% CI 1.41-1.98). Elevated rates were observed for 11 main diagnostic groups, including infections, second malignant neoplasms, and diseases of the skin, bones, and circulatory, digestive, endocrine, and urinary systems. Survivors of malignant bone tumors in childhood are at increased risk of somatic diseases many years after diagnosis. This comprehensive study contributes new insight into the risk of late effects in survivors of osteosarcoma and Ewing sarcoma, which is an essential basis for optimal patient counseling and follow-up care.

Keywords: childhood malignant bone tumors; survivorship; late effects; somatic disease; cohort study

\section{Introduction}

Osteosarcoma and Ewing sarcoma are the commonest primary malignant bone tumors in children and adolescents, accounting for approximately $6 \%$ of all childhood cancers [1,2]. The commonest site for both tumors is the extremities, but more Ewing sarcomas than osteosarcomas occur in the axial skeleton [1,2]. Chemotherapy and surgery are the primary therapy for osteosarcoma, as the tumor is not responsive to radiation at conventional doses [2]. The chemotherapeutic agents used in the treatment of osteosarcoma include cisplatin, doxorubicin, and high-dose methotrexate, and those for Ewing sarcoma are mainly vincristine, ifosfamide, doxorubicin, etoposide, actinomycin $\mathrm{D}$, and cyclophosphamide [1-3]. Ewing sarcoma is controlled locally with surgery, radiation therapy, or a combination of the two [1]. Previously, most bone tumors in the extremities were managed by amputation, however, advances in high-dose chemotherapy and in surgical techniques have made limb salvage the leading surgical procedure [2].

Survival after osteosarcoma and Ewing sarcoma diagnosed in childhood improved significantly with the introduction of chemotherapy in the 1970s, from $25 \%$ and $10 \%$ before use of chemotherapy, respectively, to 60-70\% [1,2]. Improved survival has resulted in increasing numbers of patients with long-term adverse health effects of the tumors and their treatments.

The majority of previous studies of somatic late effects in survivors of malignant bone tumors in childhood addressed either exclusively the overall risk of adverse health effects and health care use [4-6] or the risk of one or a few specific somatic late effects, especially secondary cancers [7-20]. Only a few studies [21-23] evaluated the risks of survivors of childhood malignant bone tumors for hospitalization for conditions in a wide range of main diagnostic groups, including infections, neoplasms, and diseases of the endocrine, circulatory, nervous, respiratory, digestive, and genitourinary systems. None of the studies reported risk estimates for subtypes of malignant bone tumors or for specific underlying disease categories.

In order to provide a detailed, comprehensive overview of somatic diseases that require hospital contact in long-term survivors of malignant bone tumors, we followed all children with a malignant bone tumor diagnosed in the Nordic countries. High-quality nationwide hospital registers and population registers allow long-term follow-up for medically verified diagnoses, with virtually no loss to follow-up. This is the first populationbased cohort study that provides relative rates of hospital contacts for the full range of main diagnostic groups in survivors of osteosarcoma and Ewing sarcoma and relative and absolute rates for 120 specific disease categories in survivors of childhood malignant bone tumors.

\section{Materials and Methods}

\subsection{Survivors of Malignant Bone Tumors and Comparison Cohort}

The study was conducted within the large Nordic population-based research program Adult Life after Childhood Cancer in Scandinavia (ALiCCS) [24], which comprises all children in the Nordic countries with cancer diagnosed before the age of 20 years from the 
start of the national cancer registries in the 1940s and 1950s until 2008. In accordance with the International Classifications of Childhood Cancer (ICCC) [25,26], 1661 children were registered with a primary malignant bone tumor in Denmark, Finland, Iceland, or Sweden in the ALiCCS cohort. Subsequently, 101 children were excluded because the morphology code of their tumor was not for a bone tumor or was for an unverified cancer, leaving 1560 children with a malignant bone tumor.

Since the start of population registries in the Nordic countries (Denmark, 1968; Finland, 1971; Iceland, 1955; Sweden, 1968), all residents have been assigned a unique personal identification number which allows accurate linkage of information across nationwide registers of health, migration, and vital status for all residents of the Nordic countries, with virtually no loss to follow-up.

Five comparisons for each cancer survivor were selected at random from the population registers and matched to survivors by sex, age, and country (Denmark, Finland, and Iceland) or county (Sweden). The vital and migration status of survivors and comparisons was obtained from the national population registers. Comparisons had to be alive on the date of the cancer diagnosis of the corresponding survivor and without a cancer diagnosis before 20 years of age. A total of 7796 comparisons from the general population were matched to the 1560 survivors of a malignant bone tumor. After relevant exclusions, the final dataset comprised 620 five-year survivors and 3049 comparisons (Figure 1).

In order to stratify risk estimates by the major types of bone tumor, we created two sub-cohorts consisting of survivors of osteosarcoma and of Ewing sarcoma (Supplemental Table S1, online). Topographical sites were obtained from the cancer registries and grouped into the categories "extremities" and "axial skeleton".

\subsection{Hospital Contacts for Somatic Diseases}

By linking the cohort to the national hospital registers, we obtained a full history of inpatient admissions and outpatient visits for somatic diseases ("hospital contacts"). The hospital registers contain the dates of admission and discharge, a primary discharge diagnosis, and supplementary diagnoses coded according to the International Classification of Diseases (ICD) [27-30]. Registration by treating physicians is mandatory. To estimate the disease burden among survivors, we grouped the diagnoses into 12 main groups according to the ICD, further subdivided into 120 disease categories (Supplemental Table S2, online). We included only the primary discharge diagnosis in the analyses. Information on secondary cancers in survivors and primary cancers in comparisons was obtained from the cancer registries, as the hospital registers do not distinguish between primary and secondary cancers.

\subsection{Statistical Analyses}

Follow-up started 5 years after the date of cancer diagnosis for survivors and the corresponding date for comparisons and ended at the date of death, migration, or the end of the study (Denmark, 31 October 2010; Finland, 31 December 2012; Iceland, 31 December 2008; Sweden, 31 December 2009), whichever came first. As the comparisons were restricted to individuals without a childhood cancer, follow-up for cancer (second malignant neoplasm in survivors and first primary cancer in comparisons) did not start until they were 20 years of age. Only the first diagnosis in each of the 120 disease categories was retained. As the disease categories were grouped into 12 main groups, individuals could have several diagnoses in each main group. 


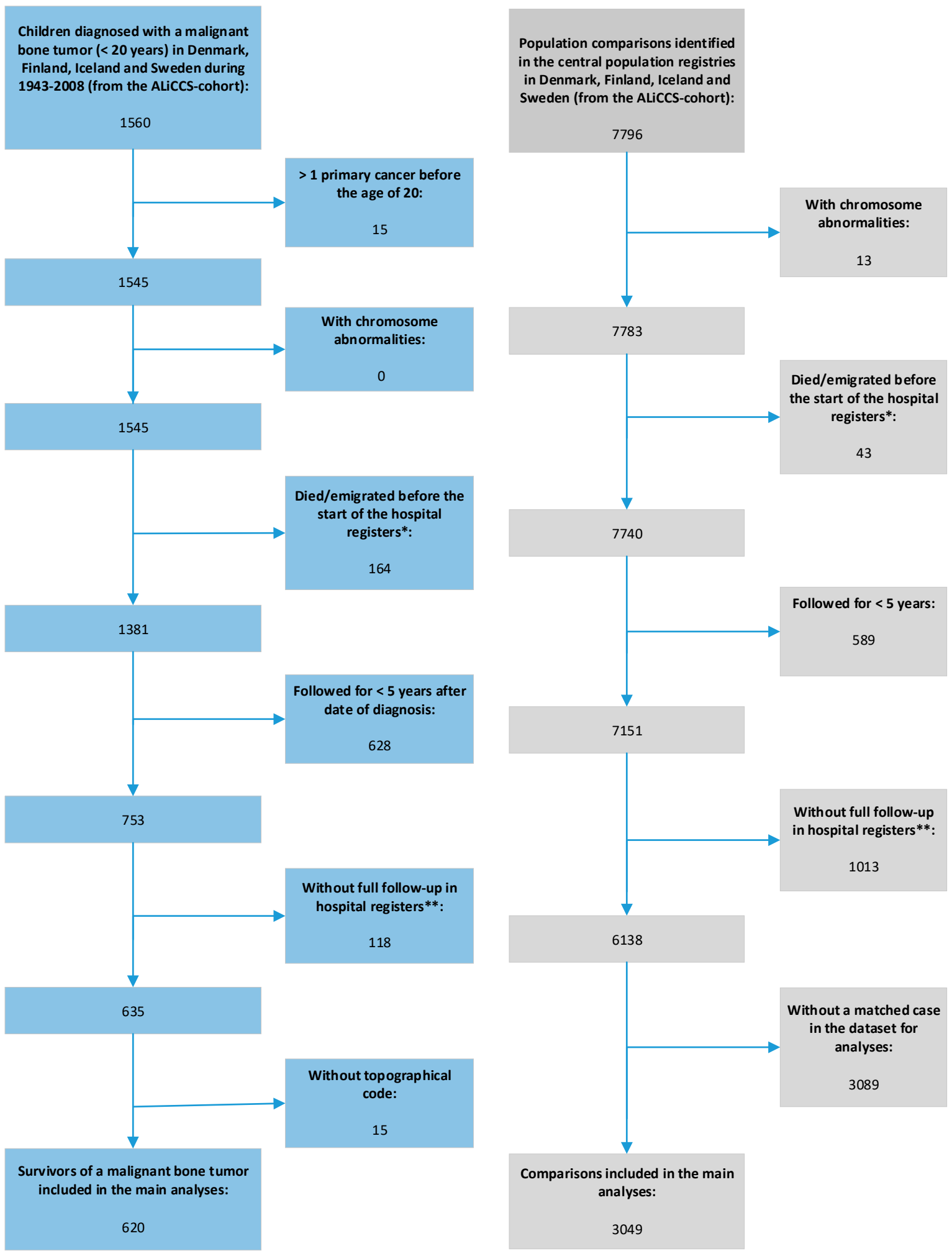

Figure 1. Flow diagram showing exclusions from the cohort of malignant bone tumor survivors and the matched comparisons cohort. * Information on inpatient admissions was available from the start of the hospital registers (Denmark, 1977; Finland, 1983; Iceland, 1999; Sweden, 1968), and outpatient visits were available for Denmark from 1995 and for Sweden from 2001. ** Individuals diagnosed more than 5 years before the start of the hospital registers were excluded to avoid a gap in information on the history of hospital contacts and to ensure accurate rates of hospital contacts. 
Rate ratios (RRs) and 95\% confidence intervals (CIs) for hospital contacts were calculated in marginal rates models [31] with age as the underlying time scale and allowing each individual to have several hospital contacts during follow-up. To account for recurrent hospital contacts for some individuals, a sandwich estimator was used for variance. We compared the overall rates of hospital contacts for any disease in the cohort of all survivors and in survivors of osteosarcoma and Ewing sarcoma with the rate in the matched comparisons. Rates were stratified by sex, age at diagnosis $(0-14 ; 15-19$ years $)$, period of diagnosis (1961-1969; 1970-1979; 1980-1989; $\geq 1990)$, years since diagnosis (5-9; 10-19; $\geq 20$ ), and cancer site (extremities, axial skeleton). RRs for hospital contacts were also calculated for the 12 main diagnostic groups. Estimates were calculated for all survivors of a malignant bone tumor and for osteosarcoma and Ewing sarcoma separately. RRs and rate differences for the 120 disease categories were calculated in unadjusted Poisson models for all survivors of a malignant bone tumor and matched comparisons.

The cumulative incidence of first hospital contact was estimated by accounting for death as a competing event and with year since diagnosis or entry as the underlying time scale. To compare the disease burden of survivors and of comparisons, we calculated the mean cumulative count of all first hospital contacts in the 120 disease categories. The cumulative incidence and the mean cumulative count were calculated for all survivors of a malignant bone tumor and for survivors of osteosarcoma and Ewing sarcoma separately.

Statistical analyses were performed in $\mathrm{R}$ version 3.6.1 and Stata version 14.2. The study was approved by the national bioethics committees, the data protection authorities, or the national institute for health and welfare in the respective countries.

\section{Results}

The survivor cohort consisted of $440(71.0 \%)$ patients with osteosarcoma and 180 (29.0\%) with Ewing sarcoma. Table 1 summarizes key characteristics of survivors and comparisons.

Table 1. Characteristics of the cohort of malignant bone tumor survivors overall, of the sub-cohorts of osteosarcoma and Ewing sarcoma, and of the matched comparison cohort.

\begin{tabular}{|c|c|c|c|c|}
\hline & \multicolumn{3}{|c|}{ 5-Year Survivors of Malignant Bone Tumors } & \multirow{2}{*}{$\begin{array}{c}\text { Comparisons } \\
\text { N (\%) }\end{array}$} \\
\hline & $\begin{array}{c}\text { All } \\
\text { N (\%) }\end{array}$ & $\begin{array}{c}\text { Osteosarcoma }{ }^{\mathrm{a}} \\
\text { N (\%) }\end{array}$ & $\begin{array}{c}\text { Ewing Sarcoma } \\
\text { N (\%) }\end{array}$ & \\
\hline Overall & $620(100.0) \mathrm{cb}$ & $440(100.0)$ & $180(100.0)$ & $3049(100.0)^{c}$ \\
\hline \multicolumn{5}{|l|}{ Country } \\
\hline Denmark and Iceland & $145(23.4)$ & $92(20.9)$ & $53(29.4)$ & $699(22.9)$ \\
\hline Finland & $176(28.4)$ & $146(33.2)$ & $30(16.7)$ & $870(28.5)$ \\
\hline Sweden & $299(48.2)$ & $202(45.9)$ & $97(53.9)$ & $1480(48.5)$ \\
\hline \multicolumn{5}{|l|}{ Period of diagnosis } \\
\hline $1961-1979$ & $129(20.8)$ & $90(20.5)$ & $39(21.7)$ & - \\
\hline 1980-1989 & $152(24.5)$ & $112(25.5)$ & $40(22.2)$ & - \\
\hline 1990-2008 & $339(54.7)$ & $238(54.1)$ & $101(56.1)$ & - \\
\hline \multicolumn{5}{|l|}{ Sex } \\
\hline Male & $338(54.5)$ & $234(53.2)$ & $104(57.8)$ & $1666(54.6)$ \\
\hline Female & $282(45.5)$ & $206(46.8)$ & $76(42.2)$ & $1383(45.4)$ \\
\hline \multicolumn{5}{|l|}{ Age at diagnosis } \\
\hline Mean (SD) & $13.7(4.0)$ & $14.2(3.7)$ & $12.3(4.4)$ & - \\
\hline $0-14$ & $347(56.0)$ & $226(51.4)$ & $121(67.2)$ & - \\
\hline $15-19$ & $273(44.0)$ & $214(48.6)$ & $59(32.8)$ & - \\
\hline \multicolumn{5}{|l|}{ Cancer site } \\
\hline Extremities & $485(78.2)$ & $382(86.8)$ & $103(57.2)$ & - \\
\hline Axial skeleton & $135(21.8)$ & $58(13.2)$ & $77(42.8)$ & - \\
\hline
\end{tabular}

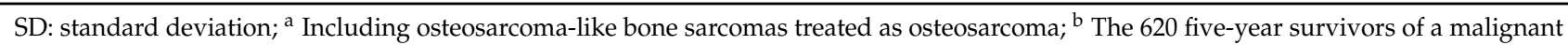
bone tumor diagnosed in childhood or adolescence were followed in the national hospital registers for a median of 12.5 years (range: 0-42 years), accruing 8854 person-years. ${ }^{c}$ The 3049 comparisons were followed-up for a median of 13.8 years (range: 0-42 years) and accrued 47,478 person-years. 
A total of 537 hospital contacts were observed among the 620 survivors of a childhood malignant bone tumor during follow-up, and survivors had an $80 \%$ higher rate of hospital contacts for any disease than comparisons (RR 1.80; 95\% CI 1.56-2.08; Table 2). After stratification by the two major types of malignant bone tumor, a statistically significantly higher rate $(p=0.044)$ was seen for survivors of Ewing sarcoma (RR 2.24; 95\% CI 1.76-2.85) than of osteosarcoma (RR 1.67; 95\% CI 1.41-1.98).

The rate of hospital contacts for any disease was higher among men $(R R=2.08)$ than women $(\mathrm{RR}=1.54$; Table 2$)$. The rate of hospital contacts remained elevated throughout follow-up, being highest $5-9$ years and $\geq 20$ years after the diagnosis.

The relative rates of hospital contacts for survivors of childhood malignant bone tumors were increased for 11 of the 12 main disease groups (statistically significantly in 10 of 12) (Figure 2). The rate of hospital contacts for respiratory diseases (RR 0.92; 95\% CI 0.68-1.22) was similar to that in matched comparisons. The highest rates were seen for diseases of the blood and blood-forming organs (RR 5.46; 95\% CI 2.20-13.59), followed by diseases of the skin and subcutaneous tissue (RR 3.57; 95\% CI 2.37-5.38) and malignant neoplasms (RR 3.47; 95\% CI 2.18-5.52). In survivors of osteosarcoma, the highest rate was seen for diseases of the skin and subcutaneous tissue (RR 3.88), followed by diseases of the blood and blood-forming organs (RR 3.30) and malignant neoplasms (RR 2.87). In survivors of Ewing sarcoma, the three highest rates were observed for diseases of the blood and blood-forming organs (RR 12.81), malignant neoplasms (RR 5.33), and diseases of the circulatory system (RR 4.22).

The RRs for hospital contacts for the specific disease categories with more than five hospital contacts observed among the survivors are shown in Figure 3. The highest relative rate was observed for heart failure (RR 16), which was observed, however, in only nine survivors (Supplemental Table S3 is a comprehensive list of numbers of hospital contacts, RRs, and 95\% CIs). Other disease categories for which the relative rate was $>5$ were sepsis, erysipelas, anemias, pericardial-, myocardial-, and endocardial disease, diseases of arteries, arterioles, and capillaries, and other disorders of the skin and subcutaneous tissue. Stratification of the overall relative rate for respiratory diseases by specific disease category showed higher rates for pneumonia but lower rates for acute upper respiratory infections and other disorders of the upper respiratory tract.

Figure 3 also provides estimates of the rate difference. None of the estimates were $>4$ per 1000 person-years. The main diagnostic group with the highest observed rate difference was diseases of bone, joint, and soft tissue, for which survivors of a malignant bone tumor had 3.98 more first hospital contacts per 1000 person-years than the comparisons. The highest rate difference of 2.38 per 1000 person-years was seen for osteomyelitis and other diseases of bone and joint.

At 25 years after diagnosis, 49.8\% (95\% CI 44.6-55.0\%) of all survivors of a malignant bone tumor and 39.3\% (95\% CI 36.9-41.6\%) of the comparisons had had at least one hospital contact for any disease (Figure 4A), and the elevated risk persisted up to 30 years after diagnosis. Stratification by type of bone tumor showed that 55.5\% of Ewing sarcoma survivors and $47.6 \%$ of osteosarcoma survivors had had at least one hospital contact for any disease 25 years after diagnosis (Figure $4 \mathrm{~B}$ ).

Figure $4 \mathrm{C}$,D shows the mean cumulative count, including all first hospital contacts for the 120 disease categories. By 25 years after diagnosis, survivors of a malignant bone tumor had had an average of 0.98 hospital contacts, and comparisons had had 0.64 . 


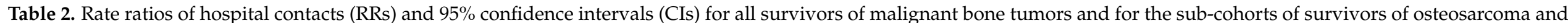
Ewing sarcoma compared with the matched comparison cohort, stratified by sex, age at diagnosis, year of diagnosis, years since diagnosis, and cancer site.

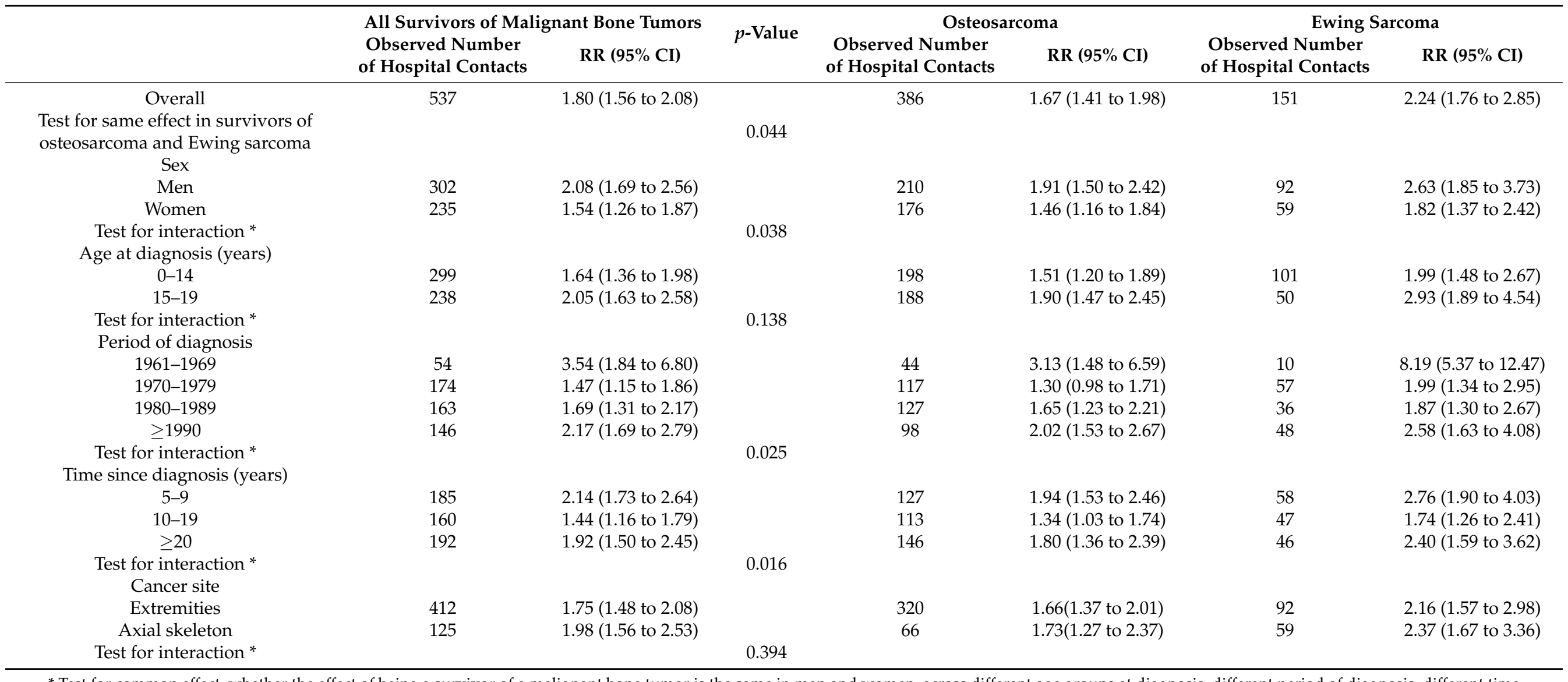

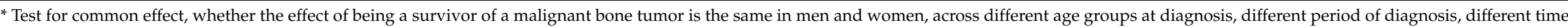
intervals since diagnosis, or in different cancer sites. 


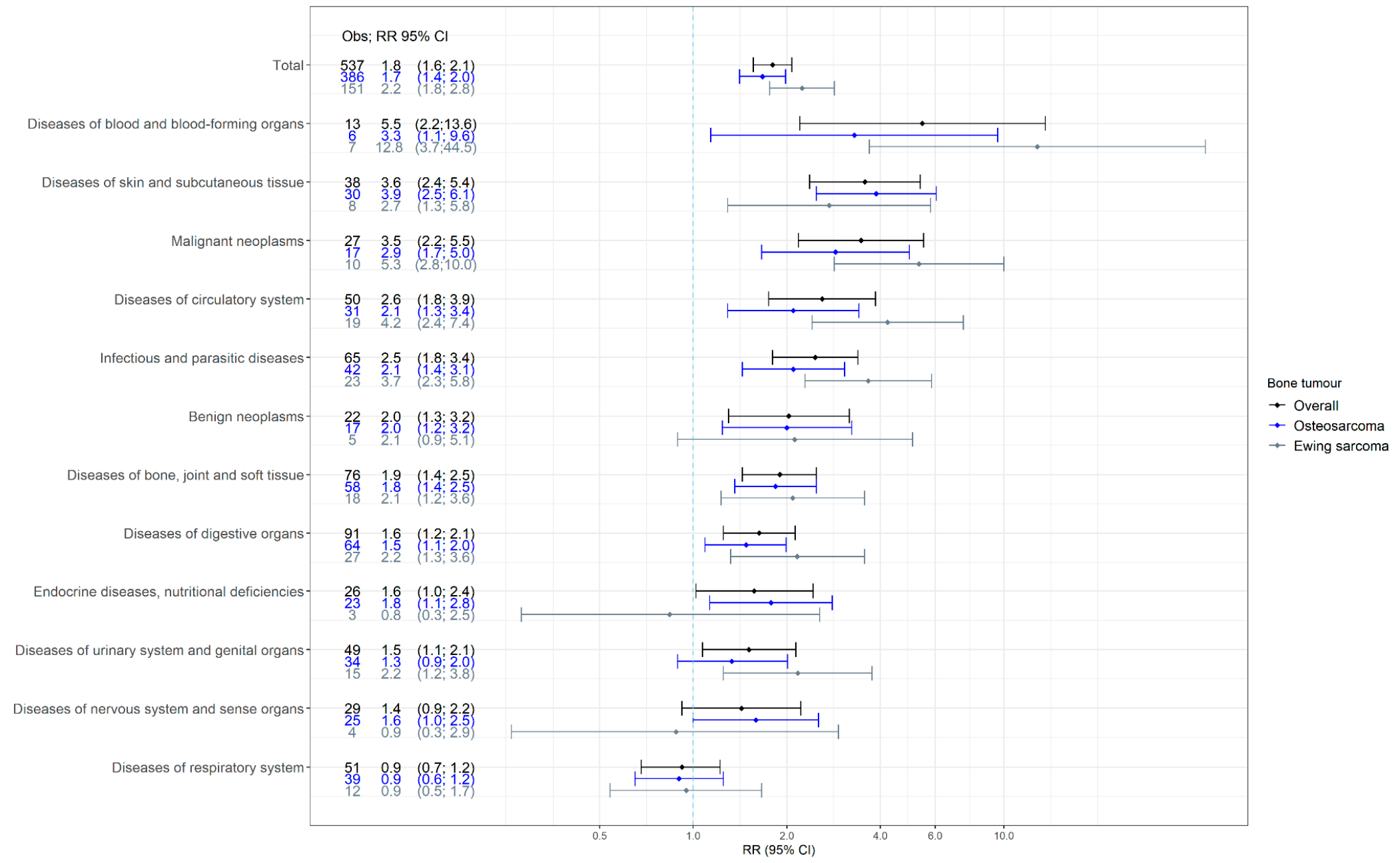

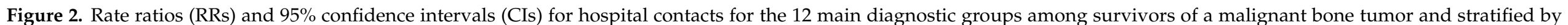
osteosarcoma and Ewing sarcoma. Obs: observed number of hospital contacts by survivors. 
Diseases of blood and blood-forming organs Diseases of skin and subcutaneous tissue Other inflammatory conditions of skin and subcutaneous tissue Other disorders of the skin and subcutaneous tissue

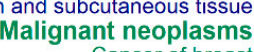
ancer of female genital organs incl. skin Infectious and parasitic diseases

$$
\begin{array}{r}
\text { Sepsis } \\
\text { Erysipelas } \\
\text { Other bacterial diseases }
\end{array}
$$

Infectious hepatitis, HIV (only in ICD-9 and 10) Other bacterial diseases Diseases of circulatory system Pericardial-, myocardial- and endocardial disease

$$
\begin{array}{r}
\text { Heart failure- } \\
\text { Conduction disorders }
\end{array}
$$
Diseases of arteries, arterioles and capillaries Venous and lymphatic disease

Diseases of bone, joint and sooft tissue Osteomyelitis and other diseases of bone and joint Other diseases of musculoskeletal system
Diseases of urinary system and genital organs Chronic cystic disease and other diseases of breast Noninflammatory disorders of female genital trac Endocrine diseases, nutritional deficiencies and other metabolic
Diseases of the thyroid gland Diabetes mellitus
Abnormal menstruation Abnormal menstruation
Diseases of digestive organs Diseases of the teeth and supporting structures
Diseases of esophagus Hernia of abdomal cavity Noninfective enteritis and colitis Other diseases of digestive syster Diseases of nervous system and sense organs Diseases of nerves and peripheral ganglia Inflammatory diseases of ear
Diseases of respiratory system Other disorders of upper respiratory tract
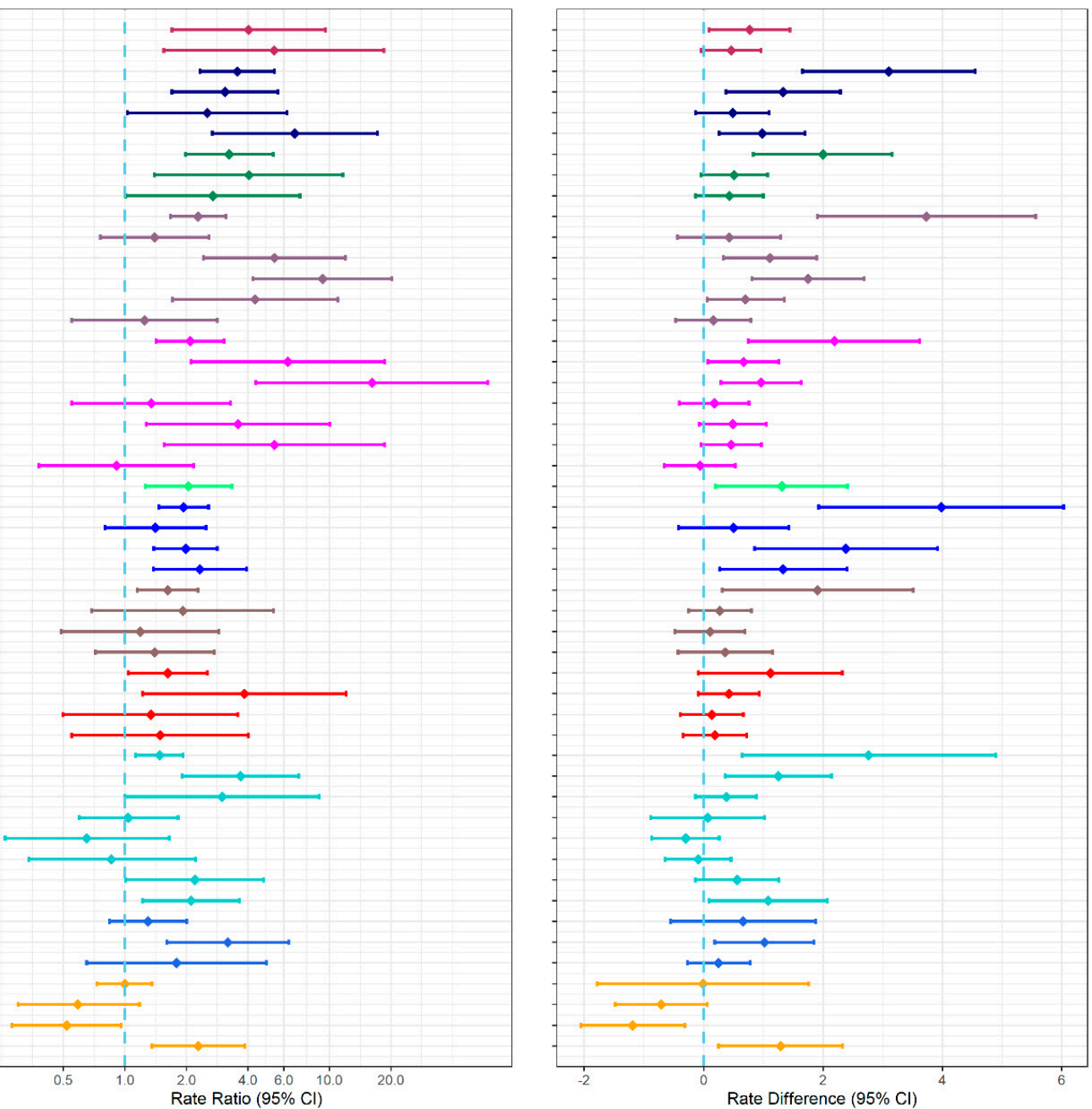

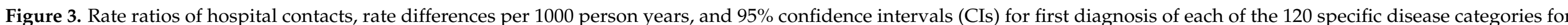
survivors compared with the matched comparisons. Only diseases for which survivors had five or more hospital contacts are shown. 


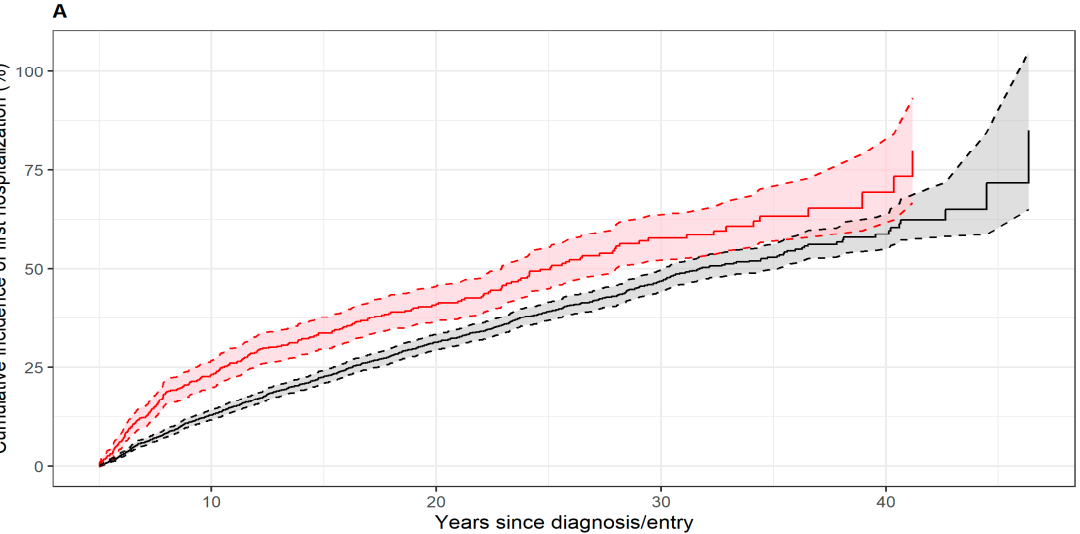

- Comparison - survivor

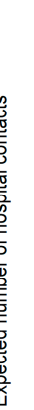

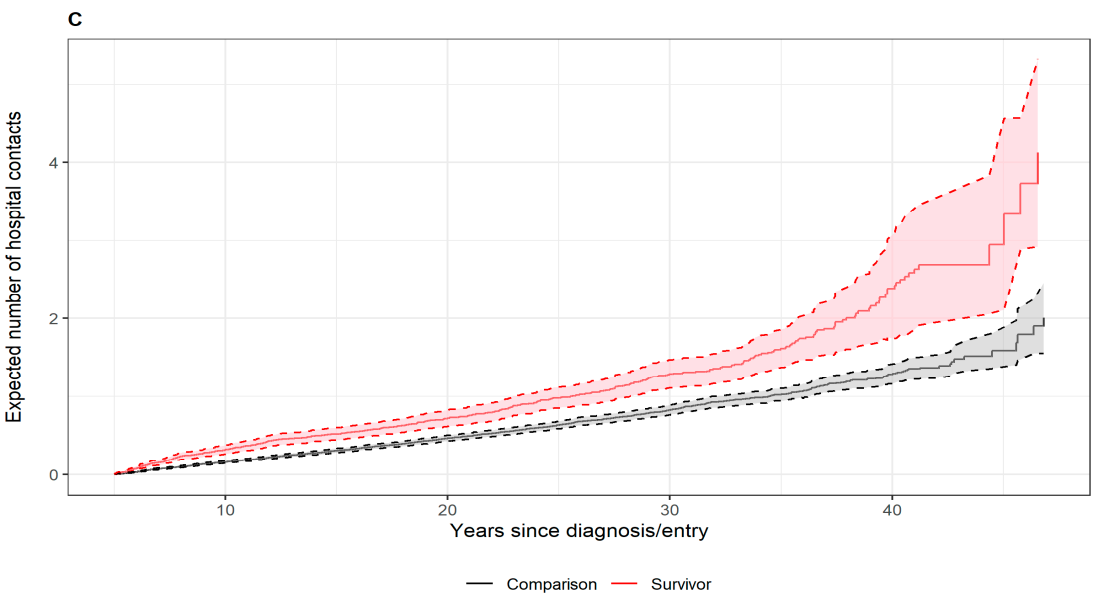

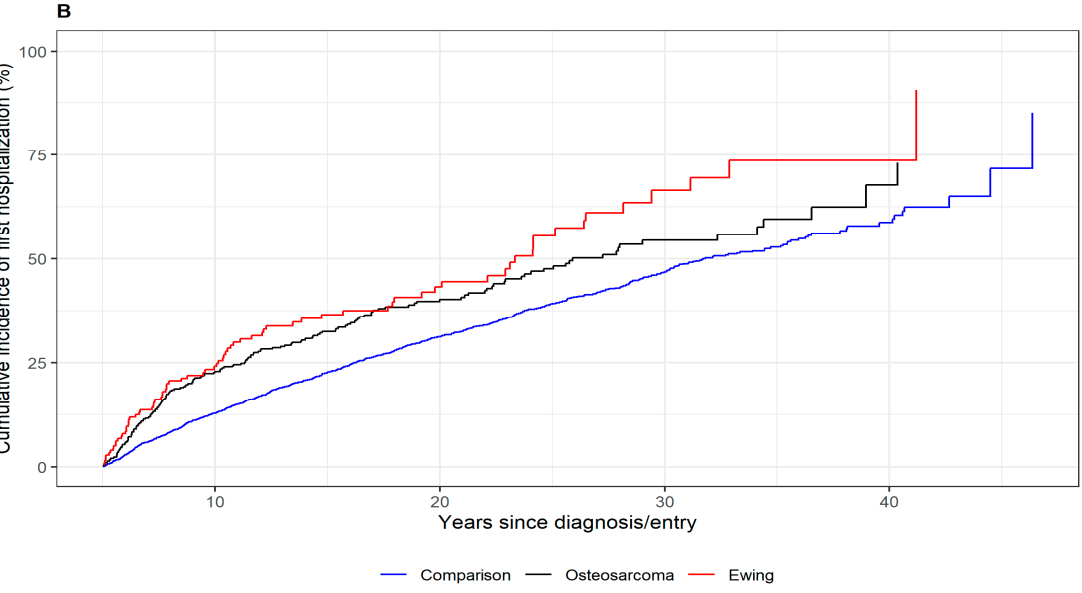

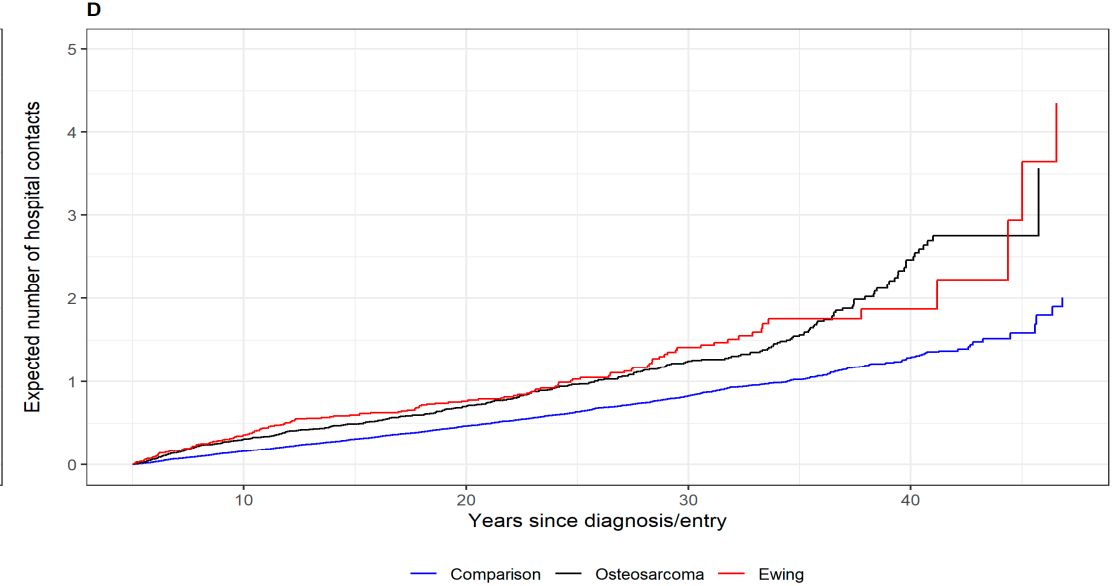

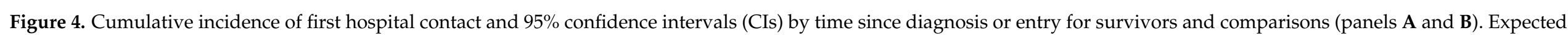
numbers of hospital contacts (mean cumulative count) with 95\% CI for survivors and their matched comparisons by time since diagnosis or entry (panels C and D). 


\section{Discussion}

This population-based cohort study of 620 survivors of a childhood malignant bone tumor in four countries showed an $80 \%$ higher rate of hospital contacts for somatic disease among survivors than matched comparisons. Survivors of Ewing sarcoma had higher rates of hospital contacts than survivors of osteosarcoma. The relative rates of hospital contacts by survivors were higher than those by comparisons for 11 of 12 main diagnostic groups.

Our finding of a 1.8-times increased risk for a hospital contact for any disease in survivors of malignant bone tumors is in line with the results of a study within the North American Childhood Cancer Survivor Study (CCSS) of self-reported hospitalization by survivors of childhood cancer, which gave a standardized incidence ratio (SIR) of 1.6 for bone cancer survivors $(n=854)$ [22]. In a cohort study of hospitalization of fiveyear survivors of childhood, adolescent, and young adult cancer in Scotland [21], the standardized hospitalization rate ratio (SHR) for 188 survivors of bone cancer was 3.8 for all diagnoses combined. The increased risks for hospital contacts for infections and diseases of the circulatory, endocrine, nervous, digestive, and genitourinary systems observed in our study are in accordance with the results of both these studies, although they reported markedly higher relative risks for second malignant neoplasms (SHR 10.3 and SIR 6.8) than the RR of 3.5 observed in our study. The lower rate of second malignant neoplasms seen in our study may be influenced by the inclusion of second malignant neoplasms occurring only after the age of 20 years.

To the best of our knowledge, this is the first population-based comparison of the risk of hospital contacts in survivors of osteosarcoma and Ewing sarcoma and to provide estimates of the relative and absolute rate of hospital contacts for 120 disease categories in survivors of malignant bone tumors. The rate of hospital contacts was higher among survivors of Ewing sarcoma than of osteosarcoma for most of the main diagnostic groups, including second malignant neoplasms, diseases of the circulatory system, infections, and diseases of the blood and blood-forming organs. The higher rates seen in survivors of Ewing sarcoma is probably due to the more frequent location in the axial skeleton and the use of radiation therapy for local control. Radiation of the chest is a well-established risk factor for cardiovascular disease [32] and for second malignancies such as sarcomas occurring in the radiation field [13].

Heart failure was the specific disease category with the highest relative rate, which is most likely attributable to the use of the cardiotoxic drug doxorubicin in the treatment of both osteosarcoma and Ewing sarcoma. The elevated risk for pericardial, myocardial, and endocardial disease is also likely to be related to doxorubicin and/or radiation therapy with the heart in the radiation field. Both limb-salvage surgery and amputation often require several surgical revisions over time to maintain a functional limb or in case of prosthesis-related infection, loosening, or breakage [1,2]. The highest rate difference was seen for osteomyelitis and other diseases of bone and joint, which is probably related to complications of endoprosthesis.

Cancer of the breast was the second malignant neoplasm with the highest relative rate in female survivors in our study, which could be related to both radiotherapy to the chest and chemotherapy. The majority of second malignancies reported in survivors of Ewing sarcoma are acute myeloid leukemia, myelodysplastic syndrome and sarcomas in the radiation field and secondary malignancies observed in osteosarcomas include leukemia, breast, lung, kidney, central nervous system, and colon cancer [2]. Most cases of leukemia in survivors of malignant bone tumors occur within five years from diagnosis [2], which explains why they were not captured in our study. The highest rate of hospital contacts of survivors was seen for diseases of the blood and blood-forming organs, especially anemias, which might be in line with the fact that hematological abnormalities in survivors of osteosarcoma have been previously documented even a long time after treatment [33].

The similar rates of hospital contacts of both survivors and comparisons for the main diagnostic group respiratory diseases were a result of a higher rate of pneumonia, but lower rates of acute upper respiratory infections and other disorders of the upper respiratory 
tract. Both the Scottish study [21] and the CCSS study [22] found elevated estimates of 2.1 and 2.9 for respiratory and pulmonary diseases but provided no information on the relative risks for specific respiratory diseases.

The major strengths of this study include the population-based design and the highquality nationwide hospital registers in the four countries, with medically verified discharge diagnoses. Moreover, as $70 \%$ of the survivors in this study who were alive five years after diagnosis survived to 40 years of age, we were able to investigate long-term somatic late effects. The matched comparison cohort was selected at random from the Nordic population registers, providing unbiased rates of hospital contacts by the general population. In addition, the survivor cohort was restricted to those with full follow-up in the national hospital registers to prevent gaps in information and ensure accurate rates of hospital contacts.

The major limitation of the study is the lack of information on treatment, which prevented investigation of the association between specific cancer treatment and risk of somatic late effects. Further, only morbidity serious enough to require a hospital contact was included, and less serious morbidity treated by a general practitioner in primary healthcare was not captured. Some of the relative risk estimates for Ewing sarcoma had high uncertainty because of the few survivors and events in this sub-group.

\section{Conclusions}

Survivors of malignant bone tumors are at increased risk of hospital contacts for somatic diseases, and the elevated risk persists up to 30 years after diagnosis. The rates of hospital contacts were increased for 11 of 12 main diagnostic groups. Survivors of Ewing sarcoma had higher rates of hospital contacts than survivors of osteosarcoma. This comprehensive population-based study provides detailed information on, and new insight into, the risk of late effects in survivors of childhood malignant bone tumors, which serves as an essential basis for patient counseling and for ensuring optimal follow-up care.

Supplementary Materials: The following are available online at https://www.mdpi.com/article/ 10.3390/cancers13184505/s1: Table S1: Grouping of malignant bone tumors in the present study; Table S2: Definition of the 12 main diagnostic groups and 120 disease categories according to the disease codes of the International Classification of Diseases, 7th to 10th revisions (ICD-7 to ICD-10); Table S3: Rate ratios (RRs) and rate differences (RDs) of hospital contact per 1000 person years and $95 \%$ confidence intervals (CIs) for first diagnosis of each of the 120 specific disease categories for survivors compared with the matched comparisons.

Author Contributions: Conceptualization, C.P., J.F.W., C.R., H.H.; methodology, C.P., J.F.W., C.R., H.H., L.K., T.B.-H. and F.N.N.; software, A.B.; formal analysis, E.A.W.A.; investigation, C.P.; data curation, J.F.W., A.B., A.S.H., L.H., L.-M.M.-H. and L.T.; writing—original draft preparation, C.P.; writing - review and editing, all authors; visualization, C.P. and E.A.W.A.; supervision, J.F.W.; project administration, C.P.; funding acquisition, C.P., J.F.W., H.H. and L.H. All authors have read and agreed to the published version of the manuscript.

Funding: This research was funded by the Danish Childhood Cancer Foundation, grant number 2014-48, 2015-32, the Swedish Childhood Cancer Fund, grant number PR2014-0121 and by the Scientific Committee of the Danish Cancer Society, grant number A7588.

Institutional Review Board Statement: The study was approved by the national bioethics committees, the data protection authorities, or the national institute for health and welfare in the respective countries.

Informed Consent Statement: Not applicable.

Data Availability Statement: Data are not available due to Danish legislation. However, the study group welcomes collaboration with other researchers using our registry data. For further information regarding collaboration, please contact Jeanette Falck Winther, (jeanette@cancer.dk). 
Conflicts of Interest: The authors declare no conflict of interest. The funders had no role in the design of the study; in the collection, analyses, or interpretation of data; in the writing of the manuscript, or in the decision to publish the results.

\section{References}

1. Heare, T.; Hensley, M.A.; Dell'Orfano, S. Bone tumors: Osteosarcoma and Ewing's sarcoma. Curr. Opin. Pediatr. 2009, 21, 365-372. [CrossRef]

2. Gorlick, R.; Perisoglou, M.; Whelan, J. Bone tumors. In Pediatric Hematology and Oncology Scientific Principles and Clinical Practice; Estlin, E., Gilbertson, R., Wynn, R., Eds.; Wiley-Blackwell: Hoboken, NJ, USA, 2010; pp. 234-257.

3. Gaspar, N.; Hawkins, D.S.; Dirksen, U.; Lewis, I.J.; Ferrari, S.; Le Deley, M.-C.; Kovar, H.; Grimer, R.; Whelan, J.; Claude, L.; et al. Ewing Sarcoma: Current Management and Future Approaches through Collaboration. J. Clin. Oncol. 2015, 33, 3036-3046. [CrossRef] [PubMed]

4. $\quad$ Fidler, M.M.; Frobisher, C.; Guha, J.; Wong, K.; Kelly, J.; Winter, D.L.; Sugden, E.; Duncan, R.; Whelan, J.; Reulen, R.C.; et al. Long-term adverse outcomes in survivors of childhood bone sarcoma: The British Childhood Cancer Survivor Study. Br. J. Cancer 2015, 112, 1857-1865. [CrossRef]

5. Kirchhoff, A.C.; Fluchel, M.N.; Wright, J.; Ying, J.; Sweeney, C.; Bodson, J.; Stroup, A.M.; Smith, K.R.; Fraser, A.; Kinney, A. Risk of hospitalization for survivors of childhood and adolescent cancer. Cancer Epidemiol. Biomark. Prev. 2014, 23, 1280-1289. [CrossRef]

6. Oeffinger, K.C.; Mertens, A.C.; Sklar, C.A.; Kawashima, T.; Hudson, M.M.; Meadows, A.T.; Friedman, D.L.; Marina, N.; Hobbie, W.; Kadan-Lottick, N.; et al. Chronic health conditions in adult survivors of childhood cancer. N. Engl. J. Med. 2006, 355, 1572-1582. [CrossRef] [PubMed]

7. Aung, L.; Gorlick, R.G.; Shi, W.; Thaler, H.; Shorter, N.A.; Healey, J.H.; Huvos, A.G.; Meyers, P.A. Second malignant neoplasms in long-term survivors of osteosarcoma: Memorial Sloan-Kettering Cancer Center Experience. Cancer 2002, 95, 1728-1734. [CrossRef] [PubMed]

8. Bacci, G.; Ferrari, C.; Longhi, A.; Ferrari, S.; Forni, C.; Bacchini, P.; Palmerini, E.; Briccoli, A.; Pignotti, E.; Balladelli, A.; et al. Second malignant neoplasm in patients with osteosarcoma of the extremities treated with adjuvant and neoadjuvant chemotherapy. $J$. Pediatr. Hematol. Oncol. 2006, 28, 774-780. [CrossRef] [PubMed]

9. Bhatia, S.; Krailo, M.D.; Chen, Z.; Burden, L.; Askin, F.B.; Dickman, P.S.; Grier, H.E.; Link, M.P.; Meyers, P.; Perlman, E.; et al. Therapy-related myelodysplasia and acute myeloid leukemia after Ewing sarcoma and primitive neuroectodermal tumor of bone: A report from the Children's Oncology Group. Blood 2007, 109, 46-51. [CrossRef] [PubMed]

10. Friedman, D.L.; Whitton, J.; Leisenring, W.; Mertens, A.C.; Hammond, S.; Stovall, M.; Donaldson, S.S.; Meadows, A.T.; Robison, L.L.; Neglia, J.P. Subsequent neoplasms in 5-year survivors of childhood cancer: The Childhood Cancer Survivor Study. J. Natl. Cancer Inst. 2010, 102, 1083-1095. [CrossRef]

11. Goldsby, R.; Burke, C.; Nagarajan, R.; Zhou, T.; Chen, Z.; Marina, N.; Friedman, D.; Neglia, J.; Chuba, P.; Bhatia, S. Second solid malignancies among children, adolescents, and young adults diagnosed with malignant bone tumors after 1976: Follow-up of a Children's Oncology Group cohort. Cancer 2008, 113, 2597-2604. [CrossRef]

12. Hamilton, S.N.; Carlson, R.; Hasan, H.; Rassekh, S.R.; Goddard, K. Long-term Outcomes and Complications in Pediatric Ewing Sarcoma. Am. J. Clin. Oncol. 2017, 40, 423-428. [CrossRef] [PubMed]

13. Kuttesch, J.F., Jr.; Wexler, L.H.; Marcus, R.B.; Fairclough, D.; Weaver-McClure, L.; White, M.; Mao, L.; Delaney, T.F.; Pratt, C.B.; Horowitz, M.E.; et al. Second malignancies after Ewing's sarcoma: Radiation dose-dependency of secondary sarcomas. J. Clin. Oncol. 1996, 14, 2818-2825. [CrossRef] [PubMed]

14. Longhi, A.; Ferrari, S.; Tamburini, A.; Luksch, R.; Fagioli, F.; Bacci, G.; Ferrari, C. Late effects of chemotherapy and radiotherapy in osteosarcoma and Ewing sarcoma patients: The Italian Sarcoma Group Experience (1983-2006). Cancer 2012, 118, 5050-5059. [CrossRef] [PubMed]

15. Navid, F.; Billups, C.; Liu, T.; Krasin, M.J.; Rodriguez-Galindo, C. Second cancers in patients with the Ewing sarcoma family of tumours. Eur. J. Cancer 2008, 44, 983-991. [CrossRef]

16. Novakovic, B.; Fears, T.R.; Horowitz, M.E.; Tucker, M.A.; Wexler, L.H. Late effects of therapy in survivors of Ewing's sarcoma family tumors. J. Pediatr. Hematol. Oncol. 1997, 19, 220-225. [CrossRef]

17. Olsen, J.H.; Moller, T.; Anderson, H.; Langmark, F.; Sankila, R.; Tryggvadottir, L.; Winther, J.F.; Rechnitzer, C.; Jonmundsson, G.; Christensen, J.; et al. Lifelong cancer incidence in 47,697 patients treated for childhood cancer in the Nordic countries. J. Natl. Cancer Inst. 2009, 101, 806-813. [CrossRef]

18. Nagarajan, R.; Kamruzzaman, A.; Ness, K.K.; Marchese, V.G.; Sklar, C.; Mertens, A.; Yasui, Y.; Robison, L.L.; Marina, N. Twenty years of follow-up of survivors of childhood osteosarcoma: A report from the Childhood Cancer Survivor Study. Cancer 2011, 117, 625-634. [CrossRef] [PubMed]

19. Ginsberg, J.P.; Goodman, P.; Leisenring, W.; Ness, K.K.; Meyers, P.A.; Wolden, S.L.; Smith, S.M.; Stovall, M.; Hammond, S.; Robison, L.L.; et al. Long-term survivors of childhood Ewing sarcoma: Report from the childhood cancer survivor study. J. Natl. Cancer Inst. 2010, 102, 1272-1283. [CrossRef]

20. Marina, N.M.; Liu, Q.; Donaldson, S.S.; Sklar, C.A.; Armstrong, G.T.; Oeffinger, K.C.; Leisenring, W.; Ginsberg, J.P.; Henderson, T.O.; Neglia, J.; et al. Longitudinal follow-up of adult survivors of Ewing sarcoma: A report from the Childhood Cancer Survivor Study. Cancer 2017, 123, 2551-2560. [CrossRef] 
21. Brewster, D.H.; Clark, D.; Hopkins, L.; Bauer, J.; Wild, S.H.; Edgar, A.B.; Wallace, W.H. Subsequent hospitalisation experience of 5-year survivors of childhood, adolescent, and young adult cancer in Scotland: A population based, retrospective cohort study. Br. J. Cancer 2014, 110, 1342-1350. [CrossRef]

22. Kurt, B.A.; Nolan, V.G.; Ness, K.K.; Neglia, J.P.; Tersak, J.M.; Hudson, M.M.; Armstrong, G.T.; Hutchinson, R.J.; Leisenring, W.M.; Oeffinger, K.C.; et al. Hospitalization rates among survivors of childhood cancer in the Childhood Cancer Survivor Study cohort. Pediatr. Blood Cancer 2012, 59, 126-132. [CrossRef] [PubMed]

23. De Fine Licht, S.; Rugbjerg, K.; Gudmundsdottir, T.; Bonnesen, T.G.; Asdahl, P.H.; Holmqvist, A.S.; Madanat-Harjuoja, L.; Tryggvadottir, L.; Wesenberg, F.; Hasle, H.; et al. Long-term inpatient disease burden in the Adult Life after Childhood Cancer in Scandinavia (ALiCCS) study: A cohort study of 21,297 childhood cancer survivors. PLoS Med. 2017, 14, e1002296. [CrossRef] [PubMed]

24. Asdahl, P.H.; Winther, J.F.; Bonnesen, T.G.; Licht, S.D.F.; Gudmundsdottir, T.; Anderson, H.; Madanat-Harjuoja, L.; Tryggvadottir, L.; Småstuen, M.C.; Holmqvist, A.S.; et al. The Adult Life after Childhood Cancer in Scandinavia (ALiCCS) Study: Design and Characteristics. Pediatr. Blood Cancer 2015, 62, 2204-2210. [CrossRef] [PubMed]

25. Birch, J.M.; Marsden, H.B. A classification scheme for childhood cancer. Int. J. Cancer 1987, 40, 620-624. [CrossRef]

26. Steliarova-Foucher, E.; Stiller, C.; Lacour, B.; Kaatsch, P. International Classification of Childhood Cancer, third edition. Cancer 2005, 103, 1457-1467. [CrossRef]

27. Ludvigsson, J.F.; Andersson, E.; Ekbom, A.; Feychting, M.; Kim, J.-L.; Reuterwall, C.; Heurgren, M.; Olausson, P.O. External review and validation of the Swedish national inpatient register. BMC Public Health 2011, 11, 450. [CrossRef]

28. Lynge, E.; Sandegaard, J.L.; Rebolj, M. The Danish National Patient Register. Scand. J. Public Health 2011, 39 (Suppl. 7), $30-33$. [CrossRef]

29. Schmidt, M.; Schmidt, S.A.; Sandegaard, J.L.; Ehrenstein, V.; Pedersen, L.; Sorensen, H.T. The Danish National Patient Registry: A review of content, data quality, and research potential. Clin. Epidemiol. 2015, 7, 449-490. [CrossRef]

30. Sund, R. Quality of the Finnish Hospital Discharge Register: A systematic review. Scand. J. Public Health 2012, 40, 505-515. [CrossRef]

31. Amorim, L.D.; Cai, J. Modelling recurrent events: A tutorial for analysis in epidemiology. Int. J. Epidemiol. 2015, 44, 324-333. [CrossRef]

32. Lipshultz, S.E.; Adams, M.J.; Colan, S.D.; Constine, L.S.; Herman, E.H.; Hsu, D.T.; Hudson, M.M.; Kremer, L.C.; Landy, D.C.; Miller, T.L.; et al. Long-term cardiovascular toxicity in children, adolescents, and young adults who receive cancer therapy: Pathophysiology, course, monitoring, management, prevention, and research directions: A scientific statement from the American Heart Association. Circulation 2013, 128, 1927-1995. [CrossRef] [PubMed]

33. Lohmann, D.J.; Hasle, H. Hematological Changes Mimicking Myelodysplastic Syndrome Following Treatment for Osteosarcoma. J. Pediatr. Hematol. Oncol. 2015, 37, 170-174. [CrossRef] 